\title{
Erratum: Half-Quantum Vortices in an Antiferromagnetic Spinor Bose-Einstein Condensate [Phys. Rev. Lett. 115, 015301 (2015)]
}

Sang Won Seo, Seji Kang, Woo Jin Kwon, and Yong-il Shin

(Received 26 January 2016; published 17 February 2016)

DOI: 10.1103/PhysRevLett.116.079901

We have found that one of the details on the spin-dependent phase-contrast imaging method is incorrectly presented in the text. The frequency of the probe light was detuned by $-20 \mathrm{MHz}$ from the $3 S_{1 / 2}|F=1\rangle \rightarrow 3 P_{3 / 2}\left|F^{\prime}=2\right\rangle$ transition, not the $3 S_{1 / 2}|F=1\rangle \rightarrow 3 P_{1 / 2}\left|F^{\prime}=2\right\rangle$ transition.

In the abstract and the text, the gapless magnon excitations associated with the observed spin fluctuations are described as transverse, but they should be referred to as axial magnons [1]. We observed no population of the $m_{z}=0$ component in the antiferromagnetic phase. This nomenclatural correction dose not affect the results and conclusion of the Letter.

[1] L. M. Symes, D. Baillie, and P. B. Blakie, Phys. Rev. A 89, 053628 (2014). 